\title{
Alectinib provides a new option for $A L K$-positive NSCLC patients after progression on crizotinib
}

See Commentary on page 239

I n December 2015, alectinib became the third ALK inhibitor approved by the United States Food and L Drug Administration for the treatment of non-smallcell lung cancer (NSCLC) that displays rearrangements of the anaplastic lymphoma kinase $(A L K)$ gene. Alectinib is a second-generation small molecule inhibitor of the ALK protein that joins ceritinib in providing a useful treatment option for patients who have progressed on crizotinib, as a result of its ability to target crizotinib-resistant mutant forms of the ALK protein. Alectinib also displays enhanced penetrance of the blood-brain barrier, which improves efficacy against central nervous system (CNS) metastases.

The FDA awarded alectinib accelerated approval on the basis of 2 phase 2, single-arm clinical trials in patients with $A L K$-positive NSCLC who had progressed on crizotinib therapy, a group of patients who have few available treatment options. Full approval is contingent upon the success of the ongoing phase 3 ALEX trial in which alectinib is being compared with crizotinib in chemotherapy-naïve patients and for which early results are anticipated in 2018.

The 2 pivotal clinical trials, NP28761 (study 1) and NP28673 (study 2), were conducted at 27 centers in the US and Canada and at 56 centers worldwide, respectively. Study 1 enrolled 87 patients and study 2138 patients aged 18 years or older, with histologically confirmed, locally advanced or metastatic $A L K$-positive NSCLC (as confirmed using an FDA-approved $A L K$ testing kit), with measurable disease according to Response Evaluation Criteria in Solid Tumors (version 1.1), and who were previously treated with crizotinib therapy. Eligible patients also had adequate hematologic, hepatic, and renal function, and an Eastern Cooperative Oncology Group performance status of 0-2. Previous chemotherapy was allowed, as were asymptomatic and neurologically unstable, untreated, or treated CNS metastases.

Patients in both studies were treated with $600 \mathrm{mg}$ oral alectinib twice daily until unacceptable toxicity or progression, and underwent tumor imaging at baseline, including computed tomography of the chest and abdomen, as well as brain imaging. Restaging scans and brain scans (for

\section{What's new, what's important}

The US Food and Drug Administration approved alectinib to treat patients with metastatic $A L K$-positive non-small-cell lung cancer whose disease had progressed or who could not tolerate treatment with crizotinib. In clinical trials, alectinib showed a partial response of $38 \%-44 \%$ and an average progression-free survival of 11.2 months. In all, $61 \%$ of patients experienced a complete or partial reduction in their brain metastatic lesions, with a progression-free survival of of 9.1 months. The recommended dose is $600 \mathrm{mg}$ orally twice daily.

The most common side effects are fatigue, constipation, swelling (edema), and muscle pain (myalgia). Treatment with alectinib may cause sunburn when patients are exposed to sunlight, and pregnant women should be notified of the possible risk to the fetus, and to use contraception throughout treatment and for at least 1 week after they receive the last dose.

There is also a warning about hepatotoxicity. Liver laboratory tests should be monitored every 2 weeks during the first 2 months of treatment, then periodically during treatment. In cases of severe elevations in alanine aminotransferase, aspartate aminotransferase, or bilirubin levels, the therapy should be withheld, then the dose reduced or permanently discontinued. This highly active drug is a very welcome addition to the treatment of $A L K$-positive lung cancer.

Jame Abraham, MD, FACP (abrahaj5@ccf.org)

patients with baseline brain metastases) were obtained at regular intervals during treatment.

The primary endpoints were objective response rate (ORR), and secondary endpoints included pharmacokinetic profile, progression-free survival (PFS), overall survival (OS), ORR in the CNS, and safety and tolerability. In both studies, the primary analysis was extended in an updated efficacy analysis. The median duration of follow-up in study NP28761 was 4.8 months in the primary analysis and 9.9 months in the updated analysis, and for NP28673, 30 weeks and 47 weeks, respectively.

Among all patients evaluable for response, ORR was $48 \%$ at primary analysis and $52 \%$ at updated analy-

Report prepared by Jane de Lartigue, PhD. JCSO 2016;14:241-243. (2016 Frontline Medical Communications. doi: 10.12788/ jeso.0277. 


\section{Mechanism of action: alectinib}

\section{Challenges of resistance and blood-brain barrier}

The anaplastic lymphoma kinase (ALK) protein is a tyrosine kinase receptor that transmits signals from the cell surface into the cell via a number of important signaling molecules, including the Ras, phosphatidylinositol 3-kinase (PI3K), phospholipase C gamma, and Janus kinase/signal transducer and activator of transcription pathways. Ultimately these signaling cascades activate the transcription of target genes in the nucleus that are involved in key cellular processes, such as proliferation and survival.

The ALK protein can become oncogenic in a number of different ways, but in $3 \%-7 \%$ of patients with non-small-cell lung cancer (NSCLC), this happens when the $A L K$ gene is broken apart and fused to another gene, the echinoderm microtubule-associated protein-like 4 gene, in a process called chromosomal translocation. This results in the expression of a fusion protein and, in this case, the fusion drives the inappropriate activation of ALK's kinase activity, which promotes cancer cell proliferation and survival.

Following the discovery of the link between $A L K$ gene rearrangements and NSCLC, small molecule inhibitors of ALK were developed in an effort to counteract the effects of oncogenic ALK. These inhibitors block the kinase activity of ALK and prevent downstream signals from being activated. Crizotinib was the first FDAapproved ALK inhibitor and proved superior to chemotherapy in the treatment of advanced $A L K$-positive NSCLC.

However, patients invariably relapse through a variety of mechanisms, including the acquisition of mutations in the ALK kinase domain that block crizotinib binding. Furthermore, secondary tumors often arise in the central nervous system where crizotinib has limited efficacy due to its poor ability to cross the blood-brain barrier.

Next-generation ALK inhibitors like alectinib have been specifically designed with the ability to target resistant forms of the ALK

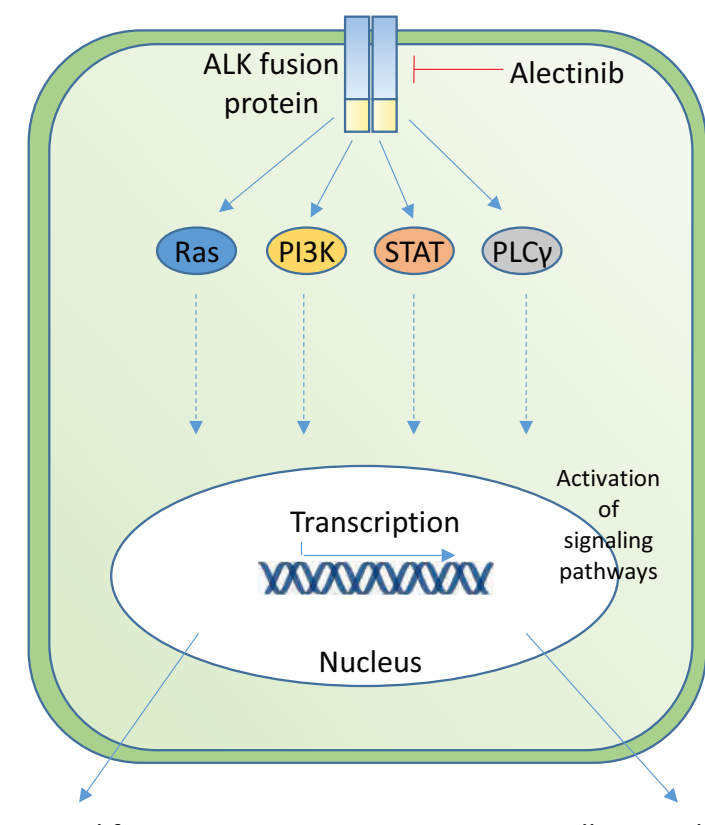

Proliferation

Cell survival

Alectinib is a small molecule inhibitor of the ALK tyrosine kinase receptor that is designed to block the activity of the wild-type ALK protein and common mutant forms that are resistant to crizotinib. It inhibits the kinase activity of the receptor and prevents activation of downstream signaling pathways, thereby blocking its effects on cancer cell proliferation and survival.

protein and with improved penetrance of the blood-brain barrier so that they might provide a treatment option beyond progression on crizotinib in this patient population. sis in study 1 ; and $49 \%$ and $50 \%$, respectively, in study 2. Median duration of response (DoR) was 13.5 months and 11.2 months in the 2 studies. Among patients with baseline CNS disease, CNS ORR was $75 \%$ in study 1 and $57 \%$ in study 2 , with median DoR of 11.2 months and 10.3 months, respectively. In those with baseline CNS metastases, a CNS complete response was achieved in $29 \%$ of patients in study 1 and in $27 \%$ of patients in study 2. A pooled analysis of the 2 studies was conducted for 51 patients with CNS metastases. ORR was $61 \%$ and CNS complete response rate was $18 \%$, with DoR of 9.1 months. In both studies, fewer CNS progression events were reported compared with non-CNS progression events.

Safety analyses were performed in 253 patients across both clinical trials, with a median duration of exposure to alectinib of 9.3 months. They indicated that adverse events (AEs) were predominantly grade 1 or 2 , most commonly constipation, fatigue, and peripheral edema. Serious AEs occurred in 19\% of patients and included pulmonary embolism, dyspnea, and hyperbilirubinemia (all 1.2\%). Fatal AEs, including hemorrhage, intestinal perforation, dyspnea, pulmonary embolism and endocarditis, occurred in $2.8 \%$ of patients overall. Alectinib was permanently discontinued as a result of AEs in 6\% of patients, most frequently due to hyperbilirubinemia and increased aspartate aminotransferase (AST) and alanine aminotransferase (ALT) levels. Dose reductions were required in $23 \%$ of patients initiating treatment at the recommended dose and median time to first dose reduction was 48 days.

The recommended dose for alectinib is $600 \mathrm{mg}$ twice daily with food, administered until disease progression or 
unacceptable toxicity. In the case of AEs, the prescribing information outlines acceptable dose reductions and recommends discontinuing treatment if patients are unable to tolerate a 300-mg dose.

Alectinib is marketed by Genentech as Alecensa. The prescribing information also details warnings and precautions relating to hepatotoxicity, interstitial lung disease/pneumonitis, bradycardia, severe myalgia, creatine phosphokinase (CPK) elevation, and embryo-fetal toxicity. During treatment with alectinib patients should have regular liver function tests, including ALT, AST, and total bilirubin every 2 weeks during the first 2 months of treatment, then periodically thereafter, and more frequently in patients who exhibit elevated levels. Based on the severity of the elevation, treatment can be withheld and resumed or permanently discontinued.

Practitioners should promptly investigate any worsening

\section{References}

1. Ou S, Ahn J, De Petris L, et al. Alectinib in crizotinib-refractory ALK-rearranged non-small-cell lung cancer: a phase 2 global study. J Clin Oncol. 2015;34:661-668.

2. Shaw AT, Gandhi L, Gadgeel S, et al. Alectinib in ALK-positive, crizotinib-resistant, non-small-cell lung cancer: a single-group, multicenter, phase 2 trial. Lancet Oncol. 2016;17:234-242.

3. Alecensa (alectinib) capsules, for oral use. Prescribing information. of respiratory symptoms that may indicate interstitial lung disease/pneumonitis and alectinib should be immediately withheld and subsequently permanently discontinued in the case of a positive diagnosis. Heart rate and blood pressure should be monitored regularly and alectinib withheld for symptomatic cases of bradycardia that is not life-threatening or permanently discontinued following recurrence or in life-threatening cases.

CPK levels should be assessed every 2 weeks for the first month and then as clinically indicated in symptomatic patients and, based on the severity of the elevation, alectinib treatment should be withheld and then resumed or the dose reduced. Patients taking alectinib are advised to avoid sun exposure and to use broad-spectrum sunscreen and pregnant women should be advised of the potential risk to a fetus and to use effective contraception during treatment and for at least 1 week after the last dose.

San Francisco, CA: Genentech USA Inc. http://www.gene.com/ download/pdf/alecensa_prescribing.pdf. Revised December 2015. Accessed March 25, 2016.

4. FDA approves new oral therapy to treat ALK-positive lung cancer [news release]. http://www.fda.gov/NewsEvents/Newsroom/ PressAnnouncements/ucm476926.htm. Released December 11, 2015. Accessed March 25, 2016. 